\title{
Enabling connectivity for tactical networks in mountainous areas by aerial relays
}

\author{
Salman M. Al-Shehri ${ }^{1} \cdot$ Pavel Loskot $^{1}$ (D) Michael J. Hirsch ${ }^{2}$
}

Published online: 24 November 2018

(c) The Author(s) 2018

\begin{abstract}
A general modeling framework for realistic performance evaluations of tactical mobile ad-hoc networks deployed in mountainous areas is presented. The framework is easily extensible, and can be eventually automated. It can be also used to generate data for other network simulators. The framework utilizes the freely downloadable high resolution 3D terrain data to define time dependent trajectories of network nodes. The node speeds and directions are linked to the terrain profile which extends the previously proposed mobility models. The path-loss analysis along the node trajectories revealed the need for aerial relays to enable full network connectivity at all times. The network consisting of 5 cluster heads and a single stationary relay is considered as a case study. The relay location and its antenna height are optimized to achieve the line-of-sight connectivity over the whole mission duration. The antenna radiation pattern at the relay is incorporated in the analysis. The resulting star network topology is used by the cluster heads to broadcast their packets to all other cluster heads. Several relaying schemes including the amplify-and-forward and the decode-and-forward relaying are studied together with the go-back-N retransmissions to achieve the reliable data transfer.
\end{abstract}

Keywords 3D terrain $\cdot$ Line-of-sight $\cdot$ MANET $\cdot$ Mobility $\cdot$ Path-loss $\cdot$ Relaying $\cdot$ Retransmission

\section{Introduction}

Mobile ad-hoc networks (MANETs) are a cornerstone of tactical communications [7]. In infrastructure-less mobile networks, the opportunities to optimize the deployment or network topology are minimum. This implies that the node connectivity is crucially affected by the availability of line-of-sight (LOS) communication links. Unfortunately, deploying tactical networks in hilly terrains and mountainous areas where the LOS visibility is easily obstructed is very common [4]. The mountains often naturally delineate borders between countries, so it is also where the conflicts most likely arise. Traditional approach for implementing the LOS connectivity is to utilize communication satellites [7]. However,

$凶$ Pavel Loskot

p.loskot@swan.ac.uk

Salman M. Al-Shehri

609122@swan.ac.uk

Michael J. Hirsch

mhirsch@iseatek.com

1 College of Engineering, Swansea University, Swansea, UK

2 ISEA TEK LLC, Maitland, FL, USA the use of satellites has a number of drawbacks. In particular, the satellites are not always visible due to the presence of high rise local obstacles such as building and hills. The orbit period of low-Earth orbit satellites is finite, so depending on the satellite network constellation, the satellite is passing above the given area once every 10's of minutes. The geo-stationary satellites are very few, and their bandwidth is shared by a number of users and communication services. This makes their usage to be very expensive. The multihop end-to-end connectivity involving satellite relays as well as large distances between the ground transceiver and the one onboard the satellite lead to very large round-trip time (RTT) delays. Moreover, the weak received signals due to large distances to satellites require specially designed bulky antennas in order to achieve acceptable data rates.

High altitude platforms (HAPs) are increasingly considered as a viable alternative to satellites [5]. The HAPs are provided by unmanned aerial vehicles (UAVs) which are normally either drones or balloons. The balloons have the advantage of being relatively cheap, easily deployable, and they can operate in very high altitudes up to the stratosphere. Their disadvantages are the limited lifetime, susceptibility to weather conditions (low pressure and temperatures, humid- 
ity, icing, lighting, and strong winds), difficulty to maintain the desired location, and relatively small payload (typically up to several kg's). Hence, the design of HAP balloons involve special materials and surface coatings as well as optimizing their aerodynamic shape.

Another option for improving the LOS visibility by raising the antennas above the ground level is to erect the antenna masts or towers. Their advantage is that they are firmly attached to the ground, so they can more easily sustain the adverse weather conditions, and multiple antennas can be mounted to achieve beamforming. The beamforming can be used to create multiple coverage sectors, suppress the interference and the jamming signals, and to direct the radiofrequency $(\mathrm{RF})$ radiation to the areas where it is needed most. However, their disadvantage is more demanding installation, limited portability, and relatively small antenna height which can be achieved (up to about $120 \mathrm{~m}$ ) compared to the other solutions.

Practical MANETs employed in military tactical missions, and also in disaster recovery scenarios require fast but temporary deployment of the supporting infrastructure. Unlike the conventional base stations which are designed to simultaneously support even 100's of subscribers, the stationary or mobile relays are added to MANETs to support the network topology by adding a small number of connections, usually between distant node clusters. Consequently, low altitude balloons (up to height of 100's $\mathrm{m}$ above the ground) appears to be one of the most viable options. Such balloons can be quickly deployed as well as removed, they are very portable, and the payload of a few kg's is sufficient to carry RF antennas with cables. Even though the primary function of the balloon relays is to enable LOS connectivity beyond local obstacles [9], they can be also utilized to offload and balance traffic in the bandwidth-limited MANETs $[10,28]$, or to provide transmission diversity [3].

In this paper, we evaluate a hypothetical tactical mission of a military squadron deployed in a mountainous area where the direct LOS connectivity is very difficult or impossible to achieve. It is shown that a single relay with balloon mounted antennas can provide sufficient LOS coverage for all the squadron platoons during the whole mission duration. In general, performance evaluations of MANETs usually assume simplified mathematical models of radiowave propagation, and focus mainly on simulating the network protocols [13]. Our approach in this paper puts emphasis on accurate modeling of radiowave propagation by assuming a realistic 3D terrain model and by assuming specific GPS (Global Positioning System) traces to emulate realistic mobility. The realistic mobility modeling in tactical networks requires good understanding of military operations and their objectives in various tactical missions. Such knowledge can be obtained, for example, from Army doctrine [4].
Our analysis exploits several freely available online tools and freely downloadable software packages, so the presented framework can be readily reproduced. The generated received signal strength (RSS) traces can be used as realistic inputs to other protocol-oriented network simulators such as NS2 [13]. The rest of this paper is organized in two main sections. In Sect. 2, we give a comprehensive description of the methodology used, and also outline the tactical mission and communication scenario considered. We explain how to create mobility traces in a realistic 3D terrain model, and investigate the corresponding radiowave propagation issues. Section 3 describes and evaluates different transmission protocols. Discussion including possible future directions is given in Sect. 4. Section 5 concludes the paper.

\subsection{Related works and technology}

Tactical networks have been the subject of research studies in many papers, although there are many more papers dealing with commercial deployment and applications of general MANETs. The connectivity aspects of tactical networks employing HAPs are investigated in the following papers. Autonomous UAVs for extending the range of tactical networks in mountainous areas are considered in [19]. The UAVs deployed in pre-selected locations can predict trajectories of mobile nodes on the ground including the expected quality of connection links in order to create the desired network topology in otherwise non-LOS environments. However, a small network of only a few nodes is considered in [19] in order to obtain tractable analysis. It is also unclear whether following rather than predicting trajectories of mobile nodes on the ground may be more beneficial. Management of such mobile tactical networks later is presented in [12].

The capacity improvement of wireless MANETs due to deployment of an airborne node is analyzed in [25]. Detailed distance-dependent channel models based on the geometric theory of diffraction are considered. It is shown that the terrain has great impact on radio propagation, and so does the altitude of airborne node on the network capacity. However, the nodes are stationary which is not realistic in tactical networks, and the airborne node is assumed to have the same design as the ground nodes.

Dynamic connectivity of MANETs with UAVs overlay assuming mobility with varying average speeds are studied in [29]. The UAVs can communicate with each other which increases the number of possible connection paths. However, the terrain profile is not considered, so all links are LOS, and all UAVs have the same altitude which is not optimized.

The paper [24] examines sectorized and non-sectorized directional antenna designs, and their impact on the link availability in networks with airborne relays. The average connectivity and link availability are evaluated as functions 
of system parameters, however, no terrain data are considered.

One of the first papers on data mulling where UAVs are used to collect data from wireless sensor nodes has been proposed in [6]. However, the sensor nodes are static, and no connectivity obstructions are assumed. The realistic design of UAV relays for tactical networks to provide beyond LOS connectivity is presented in [20]. The compatibility issues with existing and planned terrestrial and space communication networks are also considered.

A multipurpose aerial platform of UAVs for tactical surveillance networks is presented in [21] as a low-cost alternative to other solutions. It is pointed out that UAV's design must withstand all environmental and weather conditions, however, no performance evaluations were considered.

A self-organizing network of UAVs to support military operations is studied in [18]. The authors argue that it is important that UAVs are difficult to be detected, and their trajectory should not be easily predictable in order to improve their survivability and to conceal the mission objectives. Multiple trade-offs to decide on the number of UAVs to use are discussed, however, the effects of mobility and realistic radio propagation are not considered.

There are several alternatives to HAPs to provide LOS connectivity in tactical networks. For instance, radio relays can be deployed on helicopters. However, with emergence of UAVs, such solution is not economical, so it is now rarely used. The satellite relays (SATCOM) have the benefit of wide area coverage which is important especially for tactical operations at the sea. However, the bandwidth and other radio resources are rather limited, so the tactical mission objectives dictate how to assign SATCOM channels to specific military units according to operational priority.

Rapidly erected antenna towers are deployed in tactical missions when the long-term endurance is required, since it cannot be usually provided by HAPs such as UAVs or balloons. The towers are cheaper to operate, and have low maintenance requirements. Their disadvantage is relatively long deployment time, and limited flexibility in selecting the location. In addition, the tower antennas are usually at lower height above the ground compared to HAPs which may preclude LOS connectivity.

Untethered balloon relays are also common in military communications. For instance, Combat SkySat is a helium filled balloon providing UHF connectivity from above 55,000 feet, i.e., at the stratosphere. At this altitude, the practical range can reach up to 600 miles. This is particularly useful to also support aeronautical communications for aircraft in the area. The stratospheric relays are commonly used to provide wide area connectivity for long-term military missions during geopolitical conflicts.

\subsection{Paper contributions}

In our work, we assume a tethered balloon platform. Tethering stabilizes the balloon position, and provides protection against weather conditions, especially wind and lighting. Only antennas need to be attached to the balloon whereas radio heads and other equipment remain on the ground. This reduces the payload, and thus, the required size of the balloon. The main contributions of our paper can be summarized as follows.

- The aim is to produce realistic mobility traces in 3D terrain, i.e., the samples of mobile units locations at increasing time instances. Such time series data also define the movement speeds.

- The mobility traces are strongly affected by military mission objectives. In our case study, we consider reconnaissance mission of a mountainous area by several mobile units.

- We used a realistic digital elevation model (DEM) with $30 \mathrm{~m}$ resolution to construct the 3D terrain profile of a selected geographical area. The DEM data are freely available online.

- We used a free software for realistic prediction of radio propagation and radio coverage in order to optimize the balloon relay height above the ground. Consequently, we determined the minimum balloon height to achieve LOS visibility of all mobile units from the balloon at all times, and also computed the corresponding path-loss attenuations.

- We designed a simple helical antenna to be placed on the balloon, and obtained its horizontal and vertical radiation patterns.

- We found that only star topology with the balloon relay at the center is available at all times while direct connections between mobile units cannot be guaranteed.

- We designed a simple unicast protocol for sharing information packets from each mobile unit to all other mobile units. The protocol is based on orthogonal time sharing of the transmission links.

- Finally, go-back-N retransmissions are used to implement reliable packet delivery among mobile nodes. The performance of such retransmission protocol is evaluated by computer simulations.

- Overall, to our best knowledge, comprehensive frameworks such as the one presented in this paper, for realistic evaluations of performance of tactical mobile networks have not been studied in public literature previously. 


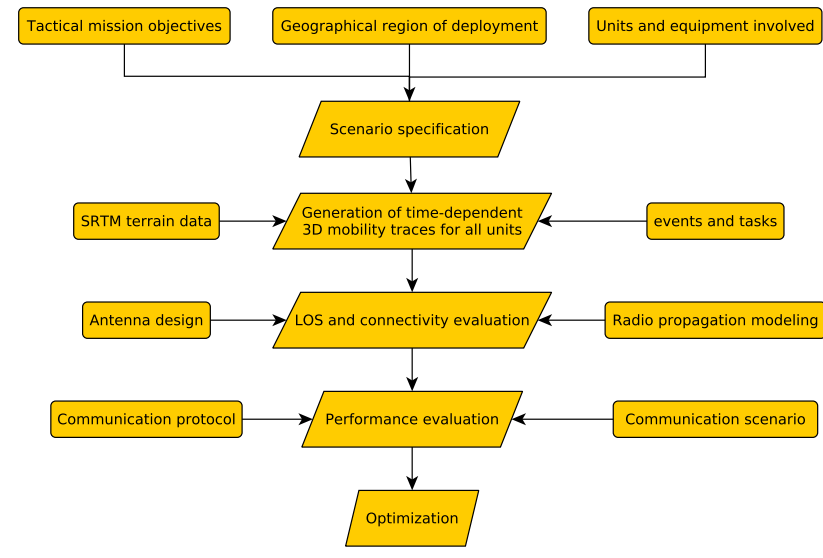

Fig. 1 The proposed framework for realistic evaluations of mobile tactical networks

\section{Modeling framework}

We present a general modeling framework to realistically evaluate the connectivity and performance of MANETs deployed in various geographical terrains. In particular, we exploit the 3D terrain profiles which have been made available through the Shuttle Radar Topography Mission (SRTM) project. The SRTM tiles are covering most of the continents with $30 \mathrm{~m}$ (1 arcsecond) resolution. The SRTM data for some very small areas (less than $0.2 \%$ ) are missing, however, the missing values can be interpolated without significant loss of accuracy. Each SRTM tile is a square matrix containing $3601 \times 3601$ elevation values encoded in 16 bits (i.e., 2 consecutive bytes) representing the average height of the $30 \mathrm{~m} \times 30 \mathrm{~m}$ cell above the mean sea level (MSL). The elevation values are expressed in meters relative to the World Geodetic System 1984 (WGS84) ellipsoid, and then adjusted to the EGM96 geoid. The data of each SRTM tile are stored in a single unique file named according to the bottom left cell of the tile. All SRTM tiles with $30 \mathrm{~m}$ resolution have been released for public use, and can be downloaded upon free registration from [26].

Our aim is to devise a modeling methodology for tactical MANETs reflecting the deployment and communication scenarios within a given geographical area. In particular, by first assuming the mission objectives, we can specify the mobility of network nodes. The mobility traces are specified as a sequence of GPS locations on the surface of the 3D terrain. Since the GPS locations are not regularly spaced, we use interpolation to obtain equidistant and more dense samples of the mobility traces. The speed of movements is assumed to be indirectly proportional to the slope of the terrain. This makes the 3D mobility traces to be time dependent. We then again use interpolation to resample the traces and obtain the 3D locations which are equally spaced at the same time instances. We note that the interpolation and (re-) sampling is done simultaneously across all mobility traces considered in order to obtain alignment of the locations in time. Next, we decide on the location of the balloon relay. The relay's location is chosen once, and then kept fixed over the mission duration in order to avoid the connectivity disruption during the relay redeployment (i.e., the relay downtime). However, the balloon height above the ground can be adaptively increased until the LOS connectivity to all nodes is achieved. By knowing the node locations at selected time instances, we can calculate the path-loss attenuations for all transmissions (i.e., links between any two nodes) considered for a given communication scenario. Since the balloon is assumed to be stationary (except the varying height), we can consider the antenna design to tilt the radiation pattern towards the moving nodes. However, the antennas carried by the mobile nodes are assumed to be omnidirectional.

The overall framework for realistic evaluation of mobile tactical networks is depicted in Fig. 1. In the first step, we need to specify the scenario to be considered including tactical mission objectives, the units and equipment to be used, and also select specific geographical area where the mission is going to be conducted. In the second step, we need to download SRTM (DEM) data, and generate a sequence of tasks and events which each tactical unit is going to encounter. This is a logical level modeling which can be conveniently done by defining model states representing tasks and events. For the generated time sequence of tasks and events, we can produce time-dependent traces of each unit. These traces usually need to be resampled, for example, in order to align the views on unit positions at the same time instances. The next step requires to assume specific antenna radiation patterns both at the HAP's relay and at each mobile unit. The antenna radiation patterns are incorporated into radio propagation models in order to test LOS connectivity and calculate the path-loss attenuations for each link at each observation time instant. The links without LOS or with path-loss attenuations larger than some pre-defined threshold can be assumed to be disconnected. In the following step, the communication network topology is used in a given communication scenario in order to evaluate the performance of a selected communication protocol. Finally, some steps can be repeated in order to perform some optimization. For example, in this paper, we optimize the HAP height above the ground level in order to provide LOS connectivity between the mobile units and the HAP relay.

More importantly, the steps just described are assumed to be independent. However, in reality, the events affect tasks, the tasks affect mobility traces, the mobility affects connectivity and network topology, the connectivity affects communication scenario, and communication scenario and topology affects the required communication protocols. Such mutual inter-dependencies may be the subject of further improving the evaluation framework for tactical mobile net- 
works, but these complications are not considered in this paper.

In the following subsections, we first describe the tactical mission and the underlying communication scenario considered. We explain in detail how to generate the time dependent 3D mobility traces. Then we design the relay, and decide on its location. Finally, we summarize the most important parameters pertaining to the modern tactical radios, and review the radio propagation models including the link budget.

\subsection{Tactical mission and communication scenario}

We assume that a military squadron of 5 platoons is deployed in a remote mountainous area. For instance, the whole unit of soldiers and equipment is air-lifted by helicopters onto a suitable planar basin. It is often the case that the destinations for each platoon as well as the paths to move to these destinations is decided beforehand unless the planning is disrupted in the course of the mission due to an unpredictable event. In the mountains, the platoons move as a group through canyons and valleys whenever possible in order to avoid steep climbs. The close proximity of soldiers within each platoon naturally creates a MANET cluster. Hence, the platoon members are likely to be within the LOS connectivity most of the time whereas the LOS connectivity among the clusters may require a high altitude balloon relay.

We assume that there are between 9 and 12 soldiers in each platoon, and every solider carries a wireless transceiver. The 5 platoons represent 5 network clusters, so the total number of nodes in the MANET is between 45 and 60. One node in each platoon is designated as a gateway or cluster head to forward traffic to other cluster heads in other platoons. On the other hand, the nodes may opportunistically overhear traffic from other nodes irrespective of their cluster membership. For simplicity, in this paper, we will consider only the first-tier sub-network, i.e., communications among the cluster heads. Moreover, since all platoons need to coordinate their activities and report to a single mission leader, we assume that all cluster heads communicate with each other and share information. Thus, the cluster heads in each platoon broadcast their traffic to all other cluster heads in other platoons. We further assume that all traffic flows are allocated the same data rate, and they are treated with the same priority. In order to combat transmission losses and guarantee the packet delivery, the packet retransmissions are also assumed.

\subsection{Mobility traces}

As a case study, a hypothetical border control tactical mission is planned within the southern mountainous part of Saudi Arabia by the Red Sea. The area is covered by the SRTM tile

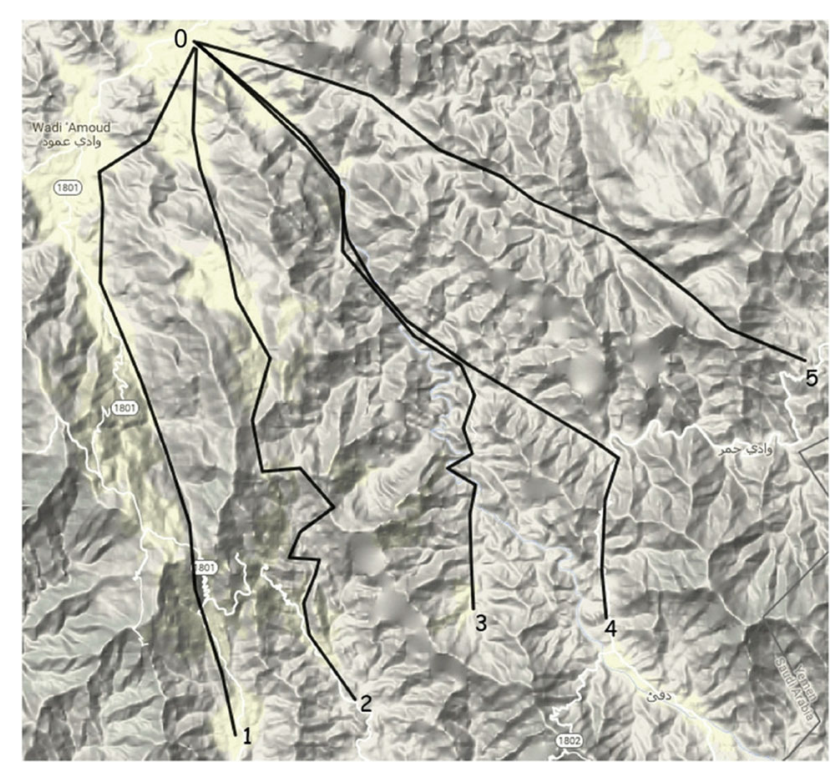

Fig. 2 The GPS defined traces of 5 platoons stemming from the common mission origin

labeled by 'N17E043'. The paths of all platoons are chosen, so that they are fully contained within the boundaries of this tile. The mission begins on a large plane (the GPS coordinates N17.397494 and E43.067436) in the mountains in the Jizan region. The 5 platoons then move in the southerly direction as shown in Fig. 2. Note that the platoons 3 and 4 follow the same trajectory for over half of their journey.

As in real-life scenarios, the platoons are trying to avoid steep climbs whenever possible, even though it may make the traveled distance longer. In order to account for a highly non-homogeneous terrain profile, the traveled path can be generated by the following procedure.

1. For a starting position $L_{0}$, randomly generate $N_{0}$ positions $L_{1}, \ldots, L_{N}$ at predetermined distance $d_{0}$ from $L_{0}$. Select $K_{0}$ out $N_{0}$ of these generated positions having the smallest elevation $\Delta h\left(L_{0}, L_{i}\right)$ and having the azimuth, $\measuredangle\left(L_{0}, L_{i}\right)$, close to the azimuth to the final destination. For each selected position, the actual distance traveled is given by the exponential distribution, $d_{i} \sim-\bar{d} \ln U$, where $\bar{d}$ is the mean distance, and $U \in(0,1)$ is the uniformly distributed random variable.

2. For each of the $K_{0}$ positions chosen in Step 1, generate $N_{1}$ new candidate positions $L$ within distance $d_{0}$. Choose $K_{1}$ out of $K_{0} N_{1}$ positions having a small elevation and a small deviation from the azimuth to the final destination. The traveled distances are again generated from the exponential distribution.

3. Repeat step 2. If the distance to the final destination of a surviving path diverges (i.e., starts increasing instead of decreasing at each step), discard this path. Alternatively, 
a replacement path can be generated by retracing back the discarded path several steps, and then generating another trajectory.

This procedure imitates a decision making process when a new direction to the destination has to be periodically selected in order to avoid obstacles, steep hills or similar.

We have used this procedure to generate the trajectories in Fig. 2 assuming the mean traveled distance $\bar{d}=1.5 \mathrm{~km}$. The trajectories were specified by a series of GPS locations using the GPS Visualizer [23]. Since the generated GPS locations defining the mobility traces are relatively farther apart, more densely spaced GPS locations are obtained by assuming linear interpolation (i.e., assuming the straight lines) in between the original locations. More precisely, we need to find the interpolated GPS locations which occur at the same instances for all 5 platoon units considered. Such interpolation is dependent on the platoon speeds, and it can also include the waiting (pausing) times. We assume that the platoon's advances are directly proportional to the inverse of the vertical steepness of the track. At the same time, it is sensible to assume that the maximum speed down the steep slopes is limited by some speed $v_{\max }$. Thus, for fixed probability of waiting $P_{\mathrm{w}}$ at a given GPS location, the speed between the two locations with the vertical separation $\Delta h$ is modeled as:

$v=\left\{\begin{array}{cl}\frac{v_{\max }}{1+\exp (\alpha \Delta h)} & \text { with probability }\left(1-P_{\mathrm{W}}\right) \\ 0 & \text { with probability } P_{\mathrm{w}}\end{array}\right.$

where $\alpha$ (in $\mathrm{s}^{-1}$ ) is a parameter adjusting the speed change over a unit time interval. Since steep mountains are unlikely to be accessible by ground vehicles, in our case study, we assume pedestrian mobility (i.e., walking soldiers) with the values $P_{\mathrm{w}}=5 \%, \alpha=0.1 \mathrm{~s}^{-1}$ and $v_{\max }=0.5 \mathrm{~m} / \mathrm{s}$ in Eq. 1 . The waiting times $T_{\mathrm{w}}$ corresponding to the zero speed are assumed to be exponentially distributed with the mean $\bar{T}_{\mathrm{w}}=$ $15 \mathrm{~min}$, so they are generated as:

$T_{\mathrm{w}} \sim-\bar{T}_{\mathrm{w}} \ln U$

where $U \in(0,1)$ is a uniformly distributed random variable. Subsequently, having adopted the speed model involving also the random waiting times, we can perform interpolation to find the GPS locations for each platoon at predetermined, regular time instances spaced $T_{s}$ minutes apart.

The trajectories sampled every $T_{s}=30 \mathrm{~min}$ are shown in Fig. 3. We can observe that the platoons traveled the distance between 23 and $26 \mathrm{~km}$. In addition, they needed between $280 \mathrm{~min}(4 \mathrm{~h} 40 \mathrm{~min})$ and $720 \mathrm{~min}(12 \mathrm{~h})$ to reach their final destinations. We can infer that, even by discounting the waiting periods, the speed of travel in the mountains is considerably dependent on the terrain profile. In such scenarios where the speed of travel as well as the direction of travel are

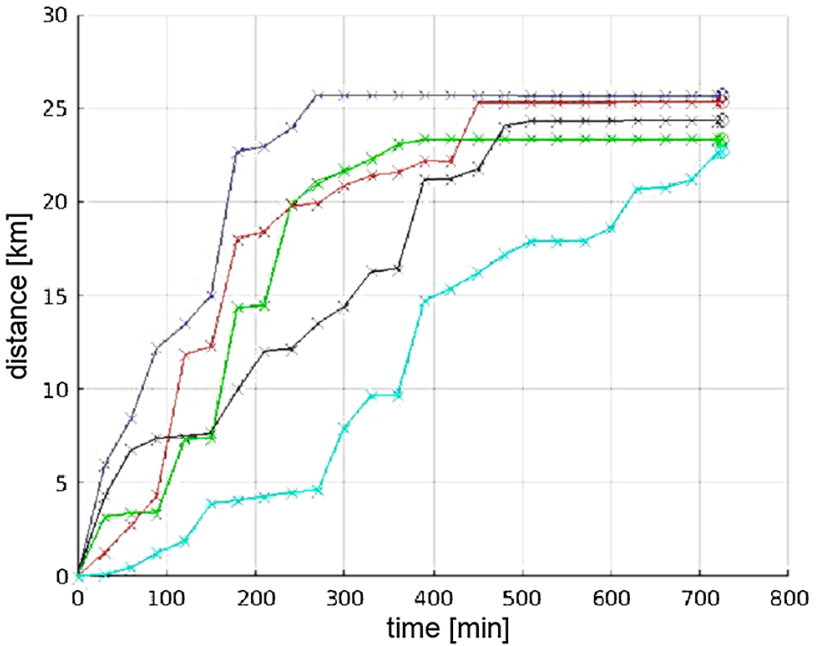

Fig. 3 The distance traveled for the 5 platoons, when the positions are sampled at $30 \mathrm{~min}$ time intervals

modulated by the terrain profile, traditional mobility models such as random waypoint mobility (RWM) and reference point group mobility (RPGM) (described, e.g., in [14]) are inadequate. The reason why these traditional mobility models fail to accurately predict the mobility in terrains with more complex 3D profile is that they prescribe the parameters of mobility implicitly assuming a flat 2D plane, so the realistic direction and speed of movements through a 3D terrain are difficult to determine.

Finally, we give 2 expressions which are used to interpolate the GPS traces. They are necessary when the traveled distances are large, and the Earth curvature cannot be neglected. The definitions can be found in documentation of [15], a small but comprehensive collection of expressions for conversions and other calculations among various coordinate systems. In particular, given the GPS coordinates lat $1, \operatorname{lon}_{1}$ ) and (lat $\left.2, \operatorname{lon}_{2}\right)$, their shortest distance $d$ over the Earth surface with the mean radius $(R)$ of $6371 \mathrm{~km}$ is given by the Haversine formulas:

$$
\begin{aligned}
& \Delta \text { lat }=\mathrm{lat}_{1}-\mathrm{lat}_{2}, \quad \Delta \mathrm{lon}=\mathrm{lon}_{1}-\mathrm{lon}_{2} \\
& a=\sin ^{2}(\Delta \mathrm{lat} / 2)+\cos \mathrm{lat}_{1} \cos \mathrm{lat}_{2} \sin ^{2}(\Delta \mathrm{lon} / 2) \\
& c=2 \operatorname{atan} 2(\sqrt{\mathrm{a}}, \sqrt{1-\mathrm{a}}), \quad \mathrm{d}=\mathrm{R} c .
\end{aligned}
$$

In addition, it is useful to determine the GPS coordinates of a point located at the given distance $d$ and bearing $\theta$ from the starting point $\left(\mathrm{lat}_{1}, \mathrm{lon}_{1}\right)$ :

$$
\begin{aligned}
\text { lat }_{2}= & \operatorname{asin}\left(\sin l_{1 a t} \cos (\mathrm{d} / \mathrm{R})+\cos \mathrm{lat}_{1} \sin (\mathrm{d} / \mathrm{R}) \cos { }^{`}\right) \\
\operatorname{lon}_{2}= & \operatorname{lon}_{1}+\operatorname{atan} 2\left(\sin ` \sin (\mathrm{d} / \mathrm{R}) \cos \operatorname{lat}_{1}, \cos (\mathrm{d} / \mathrm{R})\right. \\
& \left.-\sin \operatorname{lat}_{1} \sin \operatorname{lat}_{2}\right)
\end{aligned}
$$


Fig. 4 The platoon traces projected below the $3 \mathrm{D}$ terrain profile

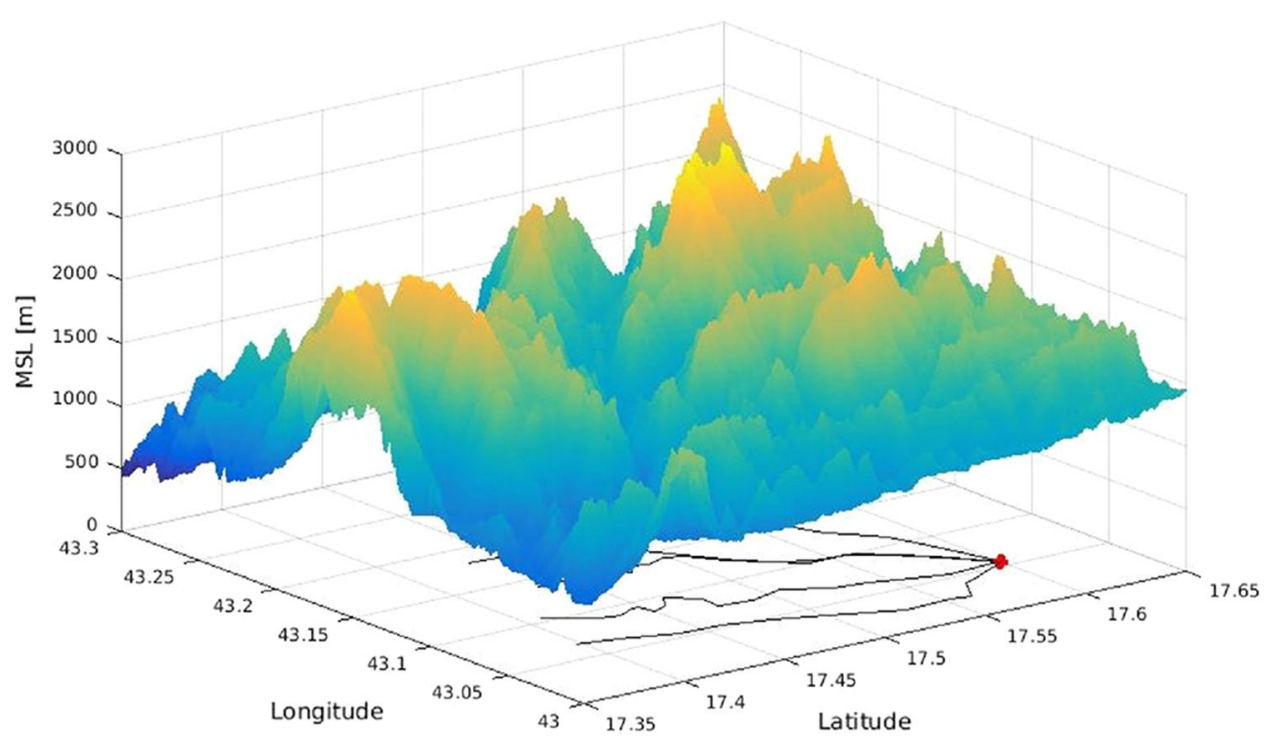

These calculations can be readily performed using the $\mathrm{C}++$ geographic library [15]. The library has bindings to (i.e., can be used with) many other programming languages.

\subsection{Relay design and deployment}

Maintaining full network connectivity in tactical networks is a mission critical requirement. The 3D terrain profile with the generated traces for 5 platoons is shown in Fig. 4. It is clear that the tall mountains prevent the LOS connectivity between the platoons most of the time. Consequently, the use of aerial platforms is necessary in order to establish the fully connected MANET.

For the mobility traces generated previously, the connectivity analysis determined that the direct LOS radio propagation paths between the platoon cluster heads are not possible for most of the time. Therefore, we deploy a single aerial relay to enable the LOS connectivity between the cluster heads and the relay, and thus, interconnect geographically dispersed clusters. The key consideration is the relay location. The distance between the relay and the cluster heads has major impact on the signal strength, and thus, also on the achievable quality-of-service (QoS), and the battery consumption of the portable wireless transceivers. On the other hand, it is desirable to deploy the relay as soon as after the mission resumes while avoiding its redeployment to maintain the cluster heads connectivity. In order to determine the most suitable location of the relay, we performed numerical experiments in the SPLAT! software. These experiments yielded the minimum required height of the relay antenna above the ground to achieve the LOS connectivity to cluster heads in all 5 platoons. An example of these measurements are given in Fig. 5. The location $L_{0}$ denotes the mission origin whereas the locations $L_{1}, \ldots, L_{5}$ have been reached exactly $2 \mathrm{~h}$ after the platoons set off on their journeys. Among these locations, the location $L_{2}$ clearly yields the smallest required antenna height of the relay to obtain th LOS connectivity. Thus, the platoon 2 would carry and deploy the relay $2 \mathrm{~h}$ after the mission beginning at the height at least $17 \mathrm{~m}$ above the ground in order to provide the LOS connectivity for the platoon number 5 . However, looking at Fig. 4, we observe that in $2 \mathrm{~h}$ the platoons traveled between 2 and almost $14 \mathrm{~km}$, respectively. It means that over these distances or over $2 \mathrm{~h}$, the connectivity among the platoons could be rather limited. On the other hand, deploying the relay at the mission origin would require placing the relay antenna at the height of at least $30 \mathrm{~m}$, but the relay would provide the desired connectivity among the platoon from the very beginning.

The platoon paths can be planned beforehand. From known high resolution $3 \mathrm{D}$ terrain maps such as those utilizing the SRTM data, it is possible to pre-calculate the optimum relay location (or several good locations) along the path of one of the platoons. Thus, there is trade-off between the deployment delay with the need to carry the relay over a certain distance and the required relay height. In the military setting, the taller the relay antennas, the easier is to intercept, jam or localize the relay transmissions. Fortunately, the carry weight of the balloon relay remains the same regardless of the height of its deployment, unlike the masses of the antenna mast or tower which are increasing with their height. Since in this paper we are assuming the balloon relay, placing the relay at the mission origin appears to be a sensible option. In this case, there is no delay in providing the LOS connectivity to the platoon cluster heads as well as no need to carry the relay to another location. Considering the mission trajectories shown in Fig. 2 and the location $L_{0}$ in Fig. 5, in the sequel, we assume that the antenna relay is lifted to $30 \mathrm{~m}$ above the ground at the point of the mission origin. 


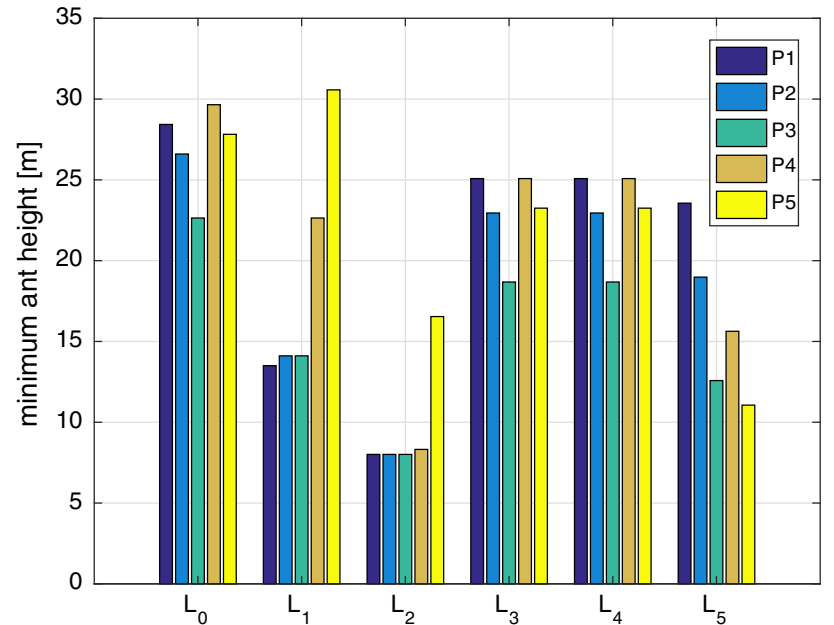

Fig. 5 The required antenna heights at 6 selected relay locations to achieve the LOS connectivity to all 5 platoon cluster heads

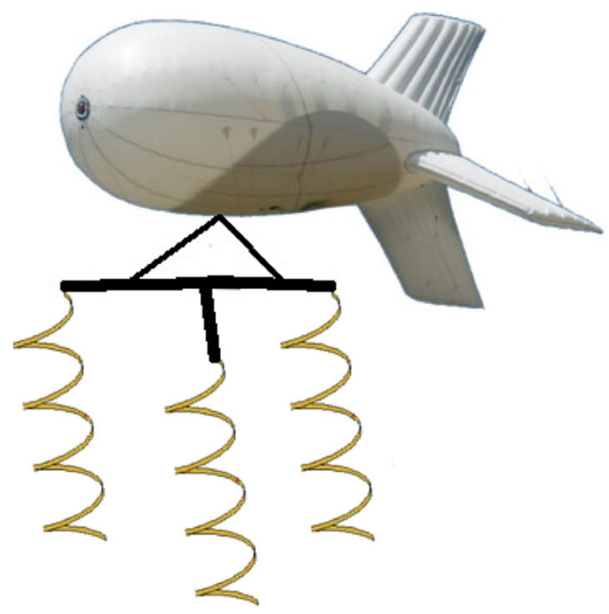

Fig. 6 The aerial relay with 3 helical diversity antennas

The balloons utilized as communication relays are usually filled with helium [8]. The balloons are anchored with wires to the ground to stabilize their position. The radio-heads with antennas are mounted on the balloon, and they are powered via the RF cable. Other wireless transceiver modules, battery and possibly a renewable energy source are left on the ground in order to minimize the balloon payload. In order to achieve a modest directivity gain while providing the wide beamwidth to achieve sufficient coverage within the desired angular sector, we can assume, for example, multiple helical antennas mounted on the balloon frame as shown in Fig. 6.

The helical antenna operated in a normal mode can satisfy our requirements for a moderate directivity gain with a wide radiation pattern. The normal mode requires that both the diameter and the length of the helix are small compared to the wavelength [27]. Moreover, the antenna can be mechanically tilted to steer the RF radiation towards the desired area. To carry out the antenna design, it is also important to decide the carrier frequency. The spectrum assignment for tactical networks is coordinated by the NATO Joint Civil/Military Frequency Agreement (NJFA). Since the very-high frequencies (VHF) are attractive due to relatively small path-loss and otherwise good propagation conditions, we assume the carrier frequency of $f_{\mathrm{c}}=44 \mathrm{MHz}$ corresponding to the wavelength $\lambda_{\mathrm{c}}=6.82 \mathrm{~m}$.

The antenna design can be carried out following mathematical expressions of electro-magnetic (EM) radiation given in books $[2,11,27]$. These expressions are already implemented in the Antenna toolbox in Matlab. In particular, we use the Antenna toolbox version 2.1 with the Matlab function dipoleHelix and the following design specification:

\begin{tabular}{ll}
\hline helix diameter: & $0.5 \mathrm{~m}$ \\
wiring width: & $0.04 \mathrm{~m}$ \\
number of turns: & 4 \\
helix spacing: & $0.5 \mathrm{~m}$ \\
feed offset from the center: & $0 \mathrm{~m}$ \\
antenna downtilt: & $15^{\circ}$ \\
\hline
\end{tabular}

It should be noted that the toolbox version matters as the previous releases may not include some antenna types or some useful functions which were added later. The radiation patterns of the resulting helix antenna can be calculated using the Matlab commands patternAzimuth and patternElevation, and displayed using the commands show, pattern, and polarplot. The radiation patterns of the designed helix antenna are shown in Fig. 7. We can observe that the directivity gain is about $1.95 \mathrm{dBi}$. In order to incorporate the horizontal and vertical antenna radiation patterns into the radio propagation model considered, both antenna patterns need to be resampled with $1^{\circ}$ resolution.

\subsection{Radio propagation}

The Longley-Rice irregular terrain model (ITM) is a widely used tool for predicting the radio wave propagation over a broad range of carrier frequencies. Recently, the Irregular Terrain With Obstructions Model (ITWOM) was introduced to improve the accuracy of the terrain diffraction calculations in the ITM. There are several software tools freely available on the Internet implementing both models. We have chosen the SPLAT! software [17], since it offers a command-line interface, and thus, is suitable for automated batch processing and optimization. In particular, we have used the SPLAT! software to determine the minimum height of the aerial relay in the 3D SRTM terrain to create a LOS visibility to all the platoon cluster heads. In the second phase, we used the SPLAT! software to accurately calculate the path-loss attenuations between the transmitting and receiving antennas. Moreover, the SPLAT! software allows to specify the 
Fig. 7 The helical antenna (a) has the 3D radiation pattern (b) and the horizontal (azimuth) and the vertical (elevation) patterns (c) and (d), respectively

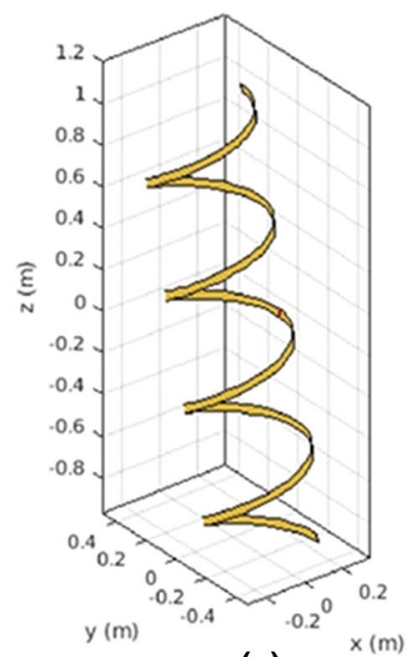

(a)

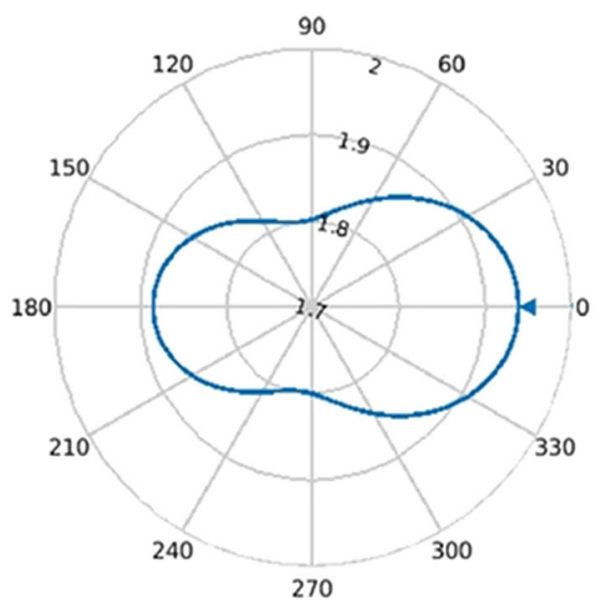

(c)

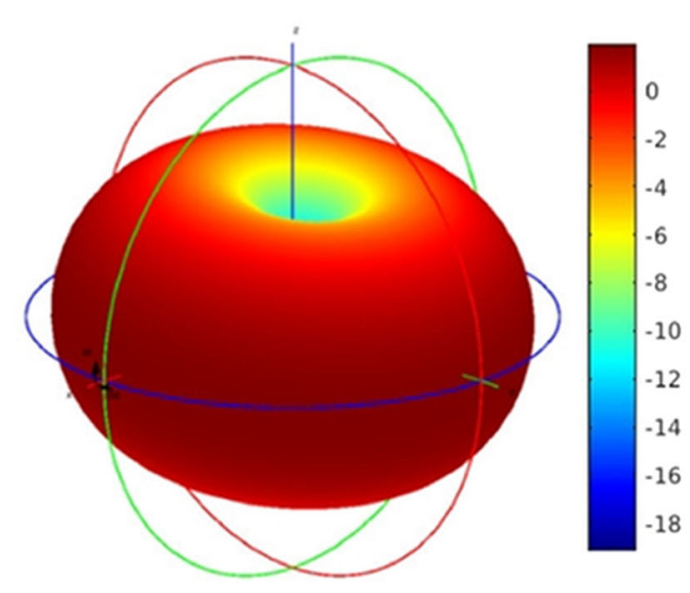

(b)

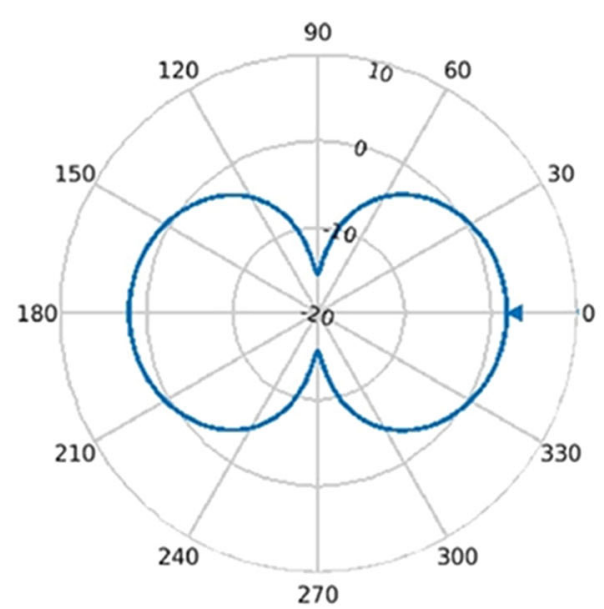

(d) horizontal and vertical antenna radiation patterns including the tilting. We used the radiation patterns in Fig. 7c, d sampled at the recommended $1^{\circ}$ resolution. The other parameters were set as follows: the carrier frequency $f_{\mathrm{c}}=44 \mathrm{MHz}$, the Earth relative permittivity 13.0 and conductivity $0.002 \mathrm{~S} / \mathrm{m}$ corresponding to the rocky and sand areas, the atmospheric bending constant $301.0 \mathrm{~N}$-Units, and the radio climate corresponding to the dessert/dry areas with high temperatures.

The SPLAT! simulations in a point-to-point mode were run in a batch using a Python script to automate the generation of the transmitter and receiver site location files. These are simple text files containing the site name, the site latitude and longitude, and the site height above the average ground level. The average ground level is used to avoid the need for interpolating DEM data points, and it is calculated as arithmetic mean of the nearby DEM data points. We confirmed that, without the aerial relay, the direct LOS visibility between the platoon cluster heads mostly does not exist (see Fig. 5). On the other hand, provided that the balloon antenna is raised to about $30 \mathrm{~m}$ above the ground whilst the cluster head antennas are kept at $1 \mathrm{~m}$ above the ground, the direct LOS connectivity between the relay and all the cluster heads becomes available at the observed GPS locations for all 5 platoon trajectories. However, it should be noted that clearing the LOS is not enough to also clear the first Fresnel zone, so there can be non-negligible multi-path propagation in addition to the LOS component. We further assume that the LOS is a dominating component of the propagation channel, and consider the calculated path-loss values shown in Fig. 8. We observe that the path-loss quickly grows to above $100 \mathrm{~dB}$, and then gradually up to $120 \mathrm{~dB}$, for all 5 platoons along their trajectories considered.

In addition to the path-loss modeling, a realistic radiowave propagation involves also the log-normally distributed shadowing and fading. The shadowing reflects the local obstacles which attenuate the transmitted signals. The fast fading can be also generated by rapid movements of the balloon antenna in the wind. Alternatively, we can account for these 


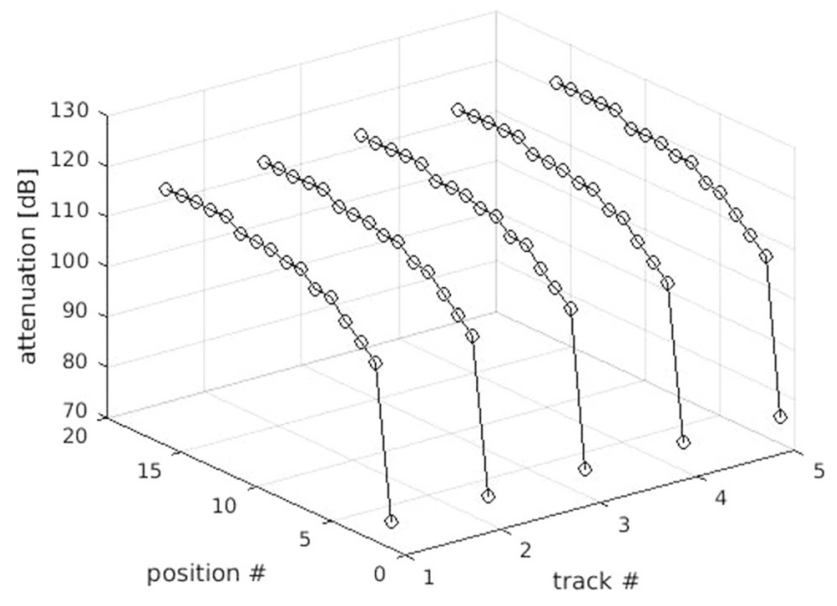

Fig. 8 The path-loss values calculated by SPLAT! for 5 platoons over their trajectories sampled at $30 \mathrm{~min}$ time intervals

other sources of signal attenuation by increasing the required signal-to-noise ratio (SNR) margins.

More importantly, as the direct communications between the cluster heads are not possible along the platoon trajectories, the cluster heads can only communicate via the relay. The resulting network topology is a star with the relay node at the center. Such topology is akin to the conventional single cell scenario with the uplink and downlink transmissions. The transmissions from the cluster heads to the relay are referred to as uplink representing a multi-access channel whereas the transmissions in the opposite direction are referred to as the downlink representing a broadcast channel. The aerial relay can assume the role of a base station. However, as discussed previously, here we assume that the relay neither acts as a data source, nor as a data sink.

\subsection{Transmission parameters}

It is useful to review the basic characteristics of military radios as they are often distinctively different from their civilian counterparts [22]. The tactical radios are normally software-configurable and software upgradable. They have multi-waveform capabilities to operate across the whole the HF/VHF/UHF frequency range at vastly different transmission powers. Furthermore, the superb receiver sensitivities must be achieved under all radio as well as environmental conditions including low and high temperatures, vibrations, dust, icing, fog and humidity. The jamming and interception resilience is achieved by narrowband signaling with the pseudo-random frequency hopping. The dimensions and weight of the wireless equipment become important when the radio units must be carried by the personnel. Furthermore, in order to ensure sufficient power supply, the soldiers normally carry several battery packs, and possibly also a portable renewable energy source.
Table 1 Link budget

\begin{tabular}{lcc}
\hline Parameter & Downlink & Uplink \\
\hline Tx power $(\mathrm{dBm})$ & $\leq 100$ & $\leq 25$ \\
Cable losses $(\mathrm{dB})$ & 3.0 & 3.0 \\
Antenna gain $(\mathrm{dBi})$ & 1.9 & 1.9 \\
Path loss $(\mathrm{dB})$ & $\leq 123$ & $\leq 123$ \\
Rx sensitivity $(\mathrm{dBm})$ & 90 & 100 \\
Noise level $(\mathrm{dBm} / \mathrm{Hz})$ & -174 & -174 \\
Bandwidth $(\mathrm{kHz})$ & 50 & 50 \\
Noise figure $(\mathrm{dB})$ & 2.0 & 1.5 \\
SNR $(\mathrm{dB})$ & $\geq 18$ & $\geq 5$ \\
\hline
\end{tabular}

The achievable data rates as well as the transmission error rates are dependent on the radio propagation conditions and the selected signaling and carrier frequency. The parameters for the link budget analysis are summarized in Table 1. We can observe that the downlink transmissions can experience about $13 \mathrm{~dB}$ larger SNR than the uplink transmissions. Hence, in general, we can expect higher data rates and more reliable transmissions in the downlink. For these SNR values, the typical packet error rates (PERs) are bounded between 2 . $10^{-1}$ and $10^{-4}$.

\section{Transmission protocols}

The SPLAT! analysis of the tactical mission in the previous section revealed the transmissions isolation in the mountainous areas. The transmissions isolation can be exploited for the interference management, especially when such phenomena can be predicted from the high resolution 3D terrain maps. The specific radiowave propagation conditions enforce certain network topology which in turn affects the transmission protocols. In this section, our objective is to evaluate protocols for exchange of information among the platoon cluster heads via the central relay. Recall that, in the communication scenario considered, all cluster heads broadcast their traffic to all other cluster heads. Note also that the PERs increase over time as the platoons move further away from the relay as indicated in Fig. 8 .

The transmissions are orthogonalized using time-division multiple access/duplex (TDMA/TDD). The TDMA/TDD together with the star topology enables straightforward network management. A fixed pre-allocation of time slots to traffic flows avoids the packet congestion and the outof-order packet delivery. Moreover, the end-to-end (e2e) management of traffic flows can be done independently. Assuming that the packet losses cannot be tolerated, some automatic request for retransmission (ARQ) scheme must be added to the protocol stack to realize the reliable data transfer 

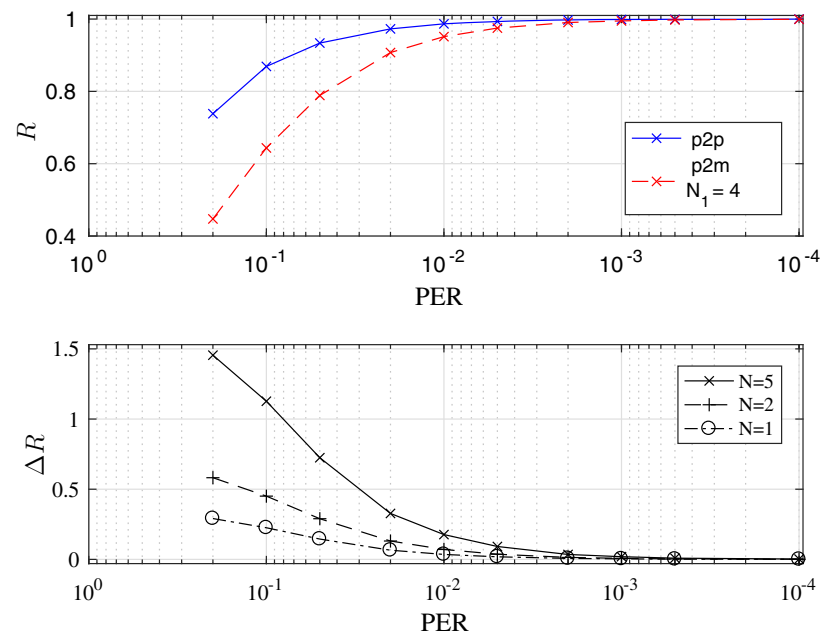

Fig. 9 The normalized packet rates for the $\mathrm{p} 2 \mathrm{p}$ and $\mathrm{p} 2 \mathrm{~m}$ GBN retransmissions (upper plot) and the normalized packet rate differences (lower plot) scaled by the total number of nodes $N$

(RDT). The ARQ acknowledgments (ACKs) are effectively multiplexed within the data packet headers, so they are also subject to losses when the erroneously received packets get discarded at the receiver. In our numerical experiments, we employ go-back-N (GBN) retransmissions [16] due to a good utilization of the channel bandwidth, and straightforward implementation. The GBN scheme does not use the receiver buffering, so out-of-order packets are discarded. This is not an issue in our network where the packets are guaranteed to be always received in order. The GBN scheme defines a finite transmission window to limit the maximum number of unacknowledged packets in the flight. The GBN acknowledgments are cumulative. The retransmissions can be also triggered by the expiry of a timer for the oldest in-flight packet; i.e., a single timer is used for a group of frames. Since the transmissions are bi-directional (to and from the other cluster heads), the expiry timers are defined at both ends of the link. For simplicity, we assume a push protocol for information delivery, in order to avoid additional complexities with the request management required by the pull information protocols. The disadvantage of using the GBN retransmissions is their much smaller efficiency when the RTT delay is large (it is not the case with a simple star topology assumed here), and when the retransmissions start to limit the desired data rate.

Consider first a general case of point-to-point (p2p) and point-to-multipoint ( $\mathrm{p} 2 \mathrm{~m})$ GBN retransmissions. The normalized packet rates measured by computer simulations are shown in Fig. 9 assuming the retransmission window size $N_{w}=10$ packets, and for the p $2 \mathrm{~m}$ scheme, the number of nodes $N=5$, i.e., the number of destination nodes, $N_{1}=N-1=4$. The simulation results in Fig. 9 assume the duality of transmission link, i.e., the PERs for data pack-
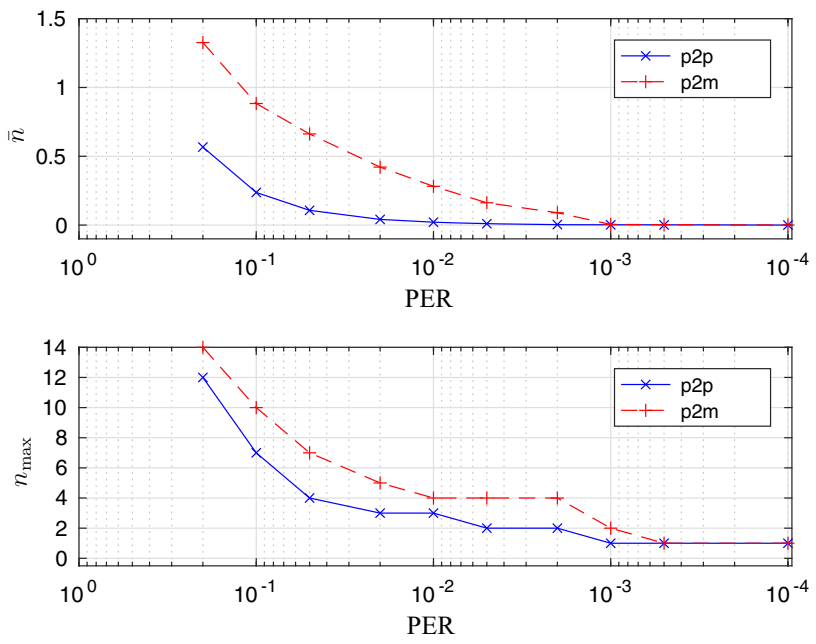

Fig. 10 The average (upper plot) and the maximum (lower plot) number of GBN retransmissions for the $\mathrm{p} 2 \mathrm{p}$ and the $\mathrm{p} 2 \mathrm{~m}$ schemes, respectively

ets in the forward link and their acknowledgments over the reverse link are equal. We observe that for the link PERs better than $10^{-3}$, the normalized packet rates of $\mathrm{p} 2 \mathrm{p}$ and $\mathrm{p} 2 \mathrm{~m}$ schemes get close to a theoretical maximum as the retransmissions become very rare. The retransmissions statistics, i.e., the mean and the maximum number of retransmissions for the GBN schemes from Fig. 9 are evaluated in Fig. 10. We again observe that the performance of both $\mathrm{p} 2 \mathrm{p}$ and $\mathrm{p} 2 \mathrm{~m}$ schemes converges to the optimum values when the PERs are better than $10^{-3}$; for these PER values, at most one retransmission is normally required.

Provided that the link PER is relatively large during the poor SNR conditions, the rate of $\mathrm{p} 2 \mathrm{~m}$ scheme can be substantially reduced. The rate loss of $\mathrm{p} 2 \mathrm{~m}$ scheme is further exacerbated with the number of nodes $N$. These issues have important consequences for the rate matching by buffering the packets at the intermediate nodes (i.e., at the relay, in our case). In particular, depending on the specific PERs of links between the relay and the cluster heads, the rate of uploading packets from the cluster head in the first hop to the relay can easily exceed the rate of reliably broadcasting the packets in the second hop from the relay to the other cluster heads. When the buffer at the relay fills up, the uplink transmissions from the cluster heads need to be halted until the downlink retransmissions again empty the relay buffer. There are also other two-hop transmission schemes for the star topology network with the relay node at the center which can avoid the rate matching and rate buffering problem at the relay as will be shown next.

In the sequel, we review 3 two-hop retransmission protocols involving different relaying strategies. In all three schemes, the relay time-synchronizes the network. The relay also adjusts the TDMA/TDD time frames to accommodate traffic requirements of the individual cluster heads. For 
instance, the relay admits, allocate and releases time slots as the cluster heads join and leave the network. The routing at the level of cluster heads with the star network topology is trivial. However, the network topology for sub-networks within the platoons with device-to-device (d2d) connectivity can be more complex, even though the number of nodes in the platoons is small (assumed between 9 and 12).

\subsection{Amplify-and-forward (AF) relaying}

The protocols involving AF relaying are usually prone to analysis as well as straightforward to implement. The relay simply retransmits the received packet in the next available time slot without checking for transmission errors. Hence, there is no need for storage buffers at the relay, and the flow control not to overwhelm the destination receiver is performed e2e at the source node. The pre-determined TDMA/TDD frame structure consists of 2 sub-frames which are further subdivided into $N$ time slots reserved for $N$ cluster head transmissions in the uplink and in the downlink, respectively. The overall packet rate can be deduced from the results in Fig. 9 by noticing that the number of retransmissions to reliably deliver the packet from the relay to all $N_{1}=N-1=4$ destination cluster heads is given by the order statistics of geometrically distributed random variables. In particular, let $K_{i}$ be the number of required retransmissions including the initial transmission attempt for the $i$-th cluster head. The random variables $K_{i}=1,2, \ldots, N_{1}$ are geometrically distributed with the cumulative density and the probability mass functions, respectively, given as:

$$
\begin{aligned}
F_{i}(k) & =\operatorname{Pr}(K \leq k)=1-P_{i}^{k} \\
f_{i}(k) & =\operatorname{Pr}(K=k)=P_{i}^{k-1}\left(1-P_{i}\right)
\end{aligned}
$$

where $P_{i}$, is the link PER which is assumed to be the same for both the uplink and the downlink. The total number of retransmissions $K$ from the relay to the $N_{1}$ cluster heads is the maximum:

$K=\max \left(K_{1}, K_{2}, \ldots, K_{N_{1}}\right)$.

Since the random variables $K_{i}$ are independent, the order statistic of maximum is:

$P_{0}\left(N_{1}\right)=\operatorname{Pr}(K=k)=\prod_{i=1}^{N_{1}} F_{i}(k)-\prod_{i=1}^{N_{1}} F_{i}(k)-f_{i}(k)$.

However, the successful packet delivery also requires its successful acknowledgment (i.e., positive ACK). The probability of packet error for the transmission of data packet in the downlink and the subsequent acknowledgment in the uplink can be represented by the overall e2e PER [3]:

$P_{\mathrm{e} 2 \mathrm{e}}=\frac{1}{2}\left(1-\left(1-2 P_{0}\left(N_{1}\right)\right)^{2}\left(1-2 P_{i}\right)^{2}\right)$.

The e2E PER 3 can be substituted for the PER (i.e., the probability of failure) $P_{i}$ used in the expression 2 to obtain the estimate of the number of $\mathrm{p} 2 \mathrm{~m}$ retransmissions which also include acknowledgments.

\subsection{Decode-and-forward (DF) relaying}

The DF relaying is more complicated to analyze than the $\mathrm{AF}$ relaying. If the relay performs the DF relaying, it is capable of immediately discarding the erroneously received packets and avoiding their subsequent broadcasting. Provided that we assume the same TDMA/TDD frame structure as for the AF relaying, i.e., there are $N$ uplink and $N$ downlink time slots allocated to $N$ network nodes, some of the downlink time slots are occasionally unused. Since the cluster heads can opportunistically listen to all downlink transmissions which is precisely the case in the communication scenario considered, the unused time slots can be shared by all traffic flows. For instance, retransmissions through unused downlink time slots can prioritize the traffic flows experiencing the worst link PER. However, our numerical experiments indicate that the improvement in the channel utilization is negligible when the link PERs are all better than $10^{-3}$.

\subsection{Independent uplink and downlink relaying}

For the AF and DF relaying schemes discussed so far, the flow control is assumed to be e2e, and all cluster heads share the TDMA/TDD frame equally. The cluster heads can send out their data with the rate of at most one packet per TDMA/TDD frame. Alternative approach is to decouple the uplink and downlink transmissions, so the flow control is done for each transmission hop independently. In particular, in the first phase, the communication channel is shared by the cluster heads in order for each to deliver $M$ packets to the relay. The mean number of transmissions of $M$ packets over the links having the PERs $P_{i}$ is:

$\bar{K}=\sum_{i=1}^{M} \mathrm{E}\left[K_{i}\right]=M \mathrm{E}\left[K_{i}\right]=M \sum_{k=0}^{\infty} k \operatorname{Pr}\left(K_{i}=k\right)$

where the probability $\operatorname{Pr}\left(K_{i}=k\right)$ is given by 2 , and $\mathrm{E}[\cdot]$ denotes the expectation. Once all successfully received packets from all cluster heads are buffered at the relay, in the second phase, the communication channel is solely used to broadcast all the $N M$ buffered packets. The probability of a given number of retransmissions of each packet to be successfully received in $N_{1}$ destinations is again given by 2 . For 
$M$ sufficiently large, the rate matching problem at the relay can be avoided. However, the transmission delay increases in proportion to the product $N \times M$, so it is less suitable for delivery of high priority traffic.

\section{Discussion}

The importance of assuming the distance dependent pathloss in performance evaluations of wireless networks is now well recognized [1]. In this paper, we extended the spatial modeling of communication networks from a $2 \mathrm{D}$ plane to the 3D terrain. The key consequence of considering the 3D terrain is the presence of natural obstacles which prevents the LOS connectivity. This may not be such a critical issue for highly optimized and pre-planned cellular networks where the base stations have enough transmission power to provide sufficient coverage even behind the obstacles. However, the deployment of MANETs is often temporary, run from batteries, and without the supporting infrastructure. Consequently, the LOS connectivity for MANETs is vital. This is evident, for example, when MANETs are used during tactical missions in the mountains and hilly terrains as demonstrated in this paper.

The satellite relays have been traditionally used to interconnect MANET segments with no LOS visibility. Although many new communication satellites are scheduled for launch in near future to provide enough capacity and coverage, the portable communication platforms such as drones and balloons operated at diverse altitudes above the ground are becoming popular, especially in military and disaster recovery scenarios. In the military operations, there is strong emphasis on mobility as well as on the environmental resilience and cyber security. In our study, we assumed only a single stationary relay with the antennas attached on a balloon. This represents a single point of failure, so it is likely to be a target of electronic warfare countermeasures. Such vulnerability can be resolved by allowing each platoon to deploy their own relay as needed. By analyzing the 3D SRTM terrain data, we observed that rising the relay antennas to 10's of meters above the ground, the LOS connectivity between platoons and the relay can be maintained at all times.

The 3D terrain model can be also used to define more realistic mobility models. Even though many mobility models have been proposed in the literature, they are mostly structural mathematical models, and they do not prescribe how to define their parameters. In this paper, we solve the problem of determining the parameters of mobility models by linking the speed and direction to the 3D terrain profile as discussed in Sect. 2.2. Our direction search algorithm is heuristic and imitate the decision making process, and it does not assume any preferred paths such as roads. The fusion of 3D terrain data and 2D maps with the existing mathematical models can be an interesting venue for the future research.

Furthermore, we could compare, for example, a stationary HAP (such as balloon in our case) with a mobile HAP represented by a single UAV (drone). However, evaluating connectivity with mobile HAP is computationally much more demanding, since the connectivity or coverage needs to be obtained for every point of the HAP trajectory. Even if we do not optimize HAP trajectory, and choose, for example, a simple circular path at some altitude, there are two parameters to optimize, i.e., the path radius and altitude. It is likely that LOS connectivity to all mobile units on the ground at all times would be obtained at higher altitude and for smaller path radius. In addition, flight durability of UAVs is often limited to 1 or $2 \mathrm{~h}$ which is not sufficient for missions lasting over $10 \mathrm{~h}$ as in our case study. Consequently, the best strategy appears to be to deploy a stationary balloon, and then also use an airborne UAV, for example, to temporarily increase capacity of some important links.

\section{Conclusion}

We have presented a comprehensive framework to study the performance of tactical networks in realistic deployment scenarios. This was accomplished by exploiting a number of freely available tools and software. Specifically, the mission objectives together with the actual 3D terrain SRTM data were used to generate time-dependent mobility traces. The LOS connectivity of the MANET was analyzed to determine the location and the antenna height of the relay, and also to calculate the path-loss attenuations. The evaluated radio propagation conditions led to a star network topology. The usefulness of the aerial relay to provide the network connectivity was clearly established. Finally, the relaying protocols with GBN retransmissions were discussed for the communication scenario considered. The future work may extend the present framework with other transmission protocols involving multiple aerial relays, antenna diversity, encryption, and $\mathrm{G} / \mathrm{M} / 1$ queuing based link control, and evaluate the network resilience against intentional jamming. Moreover, it would be very useful to automate the devised modeling framework using some programming language such as Python.

Acknowledgements S. Al-Shehri and P. Loskot were supported by Aselsan A.S., Communications and IT Division in Ankara, Turkey.

\section{Compliance with ethical standards}

Conflict of interest The authors declare that there is no conflict of interest regarding the publication of this paper.

Open Access This article is distributed under the terms of the Creative Commons Attribution 4.0 International License (http://creativecomm 
ons.org/licenses/by/4.0/), which permits unrestricted use, distribution, and reproduction in any medium, provided you give appropriate credit to the original author(s) and the source, provide a link to the Creative Commons license, and indicate if changes were made.

\section{References}

1. Alnawayseh, E. A., \& Loskot, P. (2010). Decode-and-forward cooperation as the distributed encoding and decoding. In ISWCS (pp. 656-660).

2. Balanis, C. A. (2005). Antenna theory (3rd ed.). London: Wiley.

3. Benedetto, S., \& Biglieri, E. (1999). Principles of digital transmission with wireless applications (2nd ed.). Alphen aan den Rijn: Kluwer Academic.

4. Headquarters, Department of the Army. (2007, March). Field Manual No. 3-21.8 (pp. 1-602). Washington, DC.

5. d'Oliveira, F. A., de Melo, F. C. L., \& Devezas, T. C. (2016). High-altitude platforms-Present situation and technology trends. Journal of Aerospace Technology and Management, 8, 249-262.

6. de Freitas, E. P., Heimfarth, T., Netto, I. F., Lino, C. E., Pereira, C. E., Ferreira, A. M., et al. (2010). UAV relay network to support WSN connectivity. In UMTCS (pp. 309-314).

7. Elmasry, G. F. (2012). Tactical wireless communications and networks. London: Wiley.

8. Helikite Aerostats: Technical specifications (2018). http://www. allsopp.co.uk. Accessed 9 Aug 2018.

9. Hirsch, M., \& Ortiz-Pena, H. Autonomous network connectivity and cooperative control for multiple target tracking. In Proceedings of the 27th Army Science Conference.

10. Hirsch, M. J., Sadeghnejad, A., \& Ortiz-Pena, H. (2017). Shortest paths for routing information over temporally dynamic communication networks. In Proceedings of the IEEE military communications conference (pp. 587-591).

11. Huang, Y., \& Boyle, K. (2008). Antennas. London: Wiley.

12. Hui, K. P., Pourbeik, P., George, P., Phillips, D., Magrath, S., \& Kwiatkowski, M. (2011). OPAL-A survivability-oriented approach to management of tactical military networks. In $M I L$ COM (pp. 1127-1132).

13. Issariyakul, T., \& Hossain, E. (2010). Introduction to network simulator NS2. Berlin: Springer.

14. Jayakumar, G., \& Ganapathi, G. (2008). Reference point group mobility and random waypoint models in performance evaluation of MANET routing protocols. Journal of Computer Systems, Networks, and Communications, 2008(860364), 1-10.

15. Karney, C. (2018). Geographiclib. https://geographiclib. sourceforge.io. Accessed 9 Aug 2018.

16. Kurose, J. F., \& Ross, K. W. (2012). Computer networking: A topdown approach (6th ed.). London: Pearson.

17. Magliacane, J. (2018). Signal propagation, loss, and terrain analysis tool (SPLAT!). http://www.qsl.net/kd2bd/splat.html. Accessed 9 Aug 2018.

18. Orfanus, D., de Freitas, E. P., \& Eliassen, F. (2016). Selforganization as a supporting paradigm for military UAV relay networks. IEEE Communications Letters, 20(4), 804-807.

19. Phillips, K. P. H. D., \& Kekirigoda, A. (2017). Beyond line-ofsight range extension with OPAL using autonomous unmanned aerial vehicles. In MILCOM (pp. 279-284).

20. Pinkney, M., Hampel, D., \& DiPierro, S. (1996). Unmanned aerial vehicle (UAV) communications relay. In MILCOM (pp. 47-51).

21. Rangel, R. K., de Oliveira, C. A., Kienitz, K. H., \& Brandio, M. P. (2014). Development of a multipurpose tactical surveillance system using UAV's. In Aerospace Conference (pp. 1-9).

22. Rohde\&Schwarz: Advanced HF/VHF tactical radio. (2018). Technical specifications.

23. Schneider, A. (2018). GPS Visualizer. http://www.gpsvisualizer. com/. Accessed 9 Aug 2018.

24. Shake, T., \& Amin, R. (2017). Maximizing interconnectedness and availability in directional airborne range extension networks. In MILCOM (pp. 273-278).

25. Tengstrand, S. O., Linder, S., Fors, K., \& Sterner, U. (2015). Capacity benefits of airborne nodes in ad hoc networks with broadcast traffic. In ICMCIS (pp. 1-6).

26. U.S. Geological Survey: SRTM data. (2018). https://lta.cr.usgs. gov/. See also: http://dwtkns.com/srtm30m/. Accessed 9 Aug 2018.

27. Warren, L. S. (2013). G.A.T.: Antenna theory and design (3rd ed.). London: Wiley.

28. White, K. A. (2013). Tactical network load balancing in multigateway wireless sensor nets. Ph.D. Thesis, Naval Postgraduate School.

29. Xu, Z., Huo, J., Wang, Y., Yuan, J., Shan, X., \& Feng, Z. (2011). Analyzing two connectivities in UAV-ground mobile ad hoc networks. In ICCSAE (pp. 1-5).

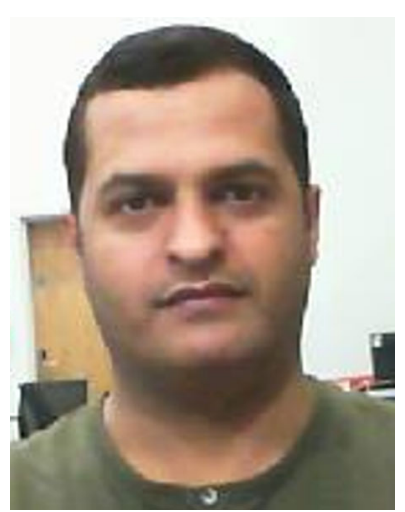

Swansea University, UK.

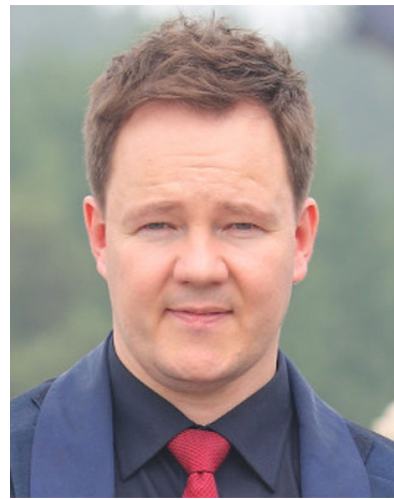

Pavel Loskot has an extensive portfolio of industrial and academic collaborative projects. $\mathrm{He}$ has been involved in telecommunication research and development since 1996. His current research is concerned with network engineering and cyber-physical system design in telecommunications, transport, and energy sectors. $\mathrm{He}$ received two best paper awards in international conferences, served as a TPC member in over 70 IEEE conferences, and delivered the keynote speeches and tutorials in a number of IEEE conferences. He is a Senior Lecturer in System Engineering at Swansea University, UK, and the Senior Member of the IEEE. 


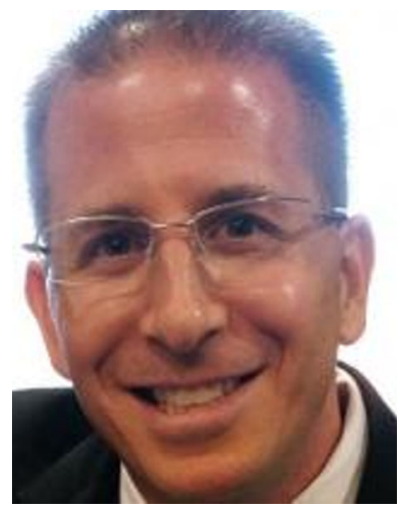

Michael J. Hirsch is the President of ISEA TEK LLC, a small women-owned business focused on delivering exceptional research and development capabilities for commercial and defense applications. He has spent the past $25+$ years developing innovative technical solutions for both defense and commercial problems. He worked for AMPAC Technologies from 1996-2001, and the Raytheon Company from 20012014. During this time, he focused on leading both small and large research teams, developing mathematical models, algorithms, prototype code, and embedded software flight code. He earned the Ph.D. degree in Systems Engineering (Operations Research concentration) from the University of Florida, a M.S. in Applied Mathematics from the University of Delaware, and a B.A. in Mathematics from West Chester University of Pennsylvania. 